\title{
ON THE LOWER CENTRAL FACTORS OF FREE CENTRE-BY-METABELIAN GROUPS
}

\author{
N. D. GUPTA, T. C. HURLEY and F. LEVIN \\ (Received 25 November 1980; revised 25 November 1982 and 25 July 1983) \\ Communicated by D. E. Taylor
}

\begin{abstract}
We describe the structure of the lower central factors of free centre-by-metabelian groups. 1980 Mathematics subject classification (Amer. Math. Soc.): 20 E 05, 20 E 10, 20 F 12, 20 F 14.
\end{abstract}

\section{Introduction}

The lower central factors of free polynilpotent groups are torsion-free (Šmelkin [7], Ward [8]), but the $c$ th lower central factor $\Phi_{c}\left(G_{n}\right)$ of a free centre-by-metabelian group $G_{n}$ of rank $n \geqslant 2$ may have 2-torsion for $c \geqslant 5$. This was first shown by Ridley [6] for $n=2, c$ even, and later for $n \geqslant 5, c$ odd, by Hurley (unpublished). For $c \leqslant 4, \Phi_{c}\left(G_{n}\right)$ is the same as the corresponding factor of a free group of rank $n$.

In this paper, we give an explicit basis for $\Phi_{c}\left(G_{n}\right)$. Gupta and Levin [2] observed that the structure of the torsion group $T_{n, c}$ of $\Phi_{c}\left(G_{n}\right)$ varies drastically according to whether $c$ is even or odd, but in either case $T_{n, c}$ is an elementary abelian 2-group.

\section{Notation}

Our commutator notation is $\left[g_{1}, g_{2}\right]=g_{1}^{-1} g_{2}^{-1} g_{1} g_{2},\left[g_{1}, \ldots, g_{n+1}\right]=\left[\left[g_{1}, \ldots, g_{n}\right]\right.$, $\left.g_{n+1}\right]$ and $\left[g_{1}, g_{2} ; g_{3}, g_{4}\right]=\left[\left[g_{1}, g_{2}\right],\left[g_{3}, g_{4}\right]\right]$ for group elements, with analogous

○ 1985 Australian Mathematical Society 0263-6115/85\$A2.00+0.00 
notation for the ring commutator $\left(r_{1}, r_{2}\right)=r_{1} r_{2}-r_{2} r_{1}$. further, $\left[g_{1}, k g_{2}\right]=$ $\left[g_{1}, g_{2}, \ldots, g_{2}\right], k$ repeats of $g_{2},\left(\left[g_{1}, 0 g_{2}\right]=g_{1}\right)$. For $n \geqslant 1, \gamma_{n}(G)$ is the $n$th term of the lower central series of $G, G^{\prime}=\gamma_{2}(G), G^{\prime \prime}=\gamma_{2}\left(G^{\prime}\right)$. All other notation follows Hanna Neumann [5].

\section{Preliminaries}

Much of the technical work required in this paper has been carried out in [2]. As in [2], the main tool in our investigation is the following power series representation (cf. [3]). Let $\left.\mathbf{P}_{n}=\mathbf{Z}\left[y_{1}, \ldots, y_{n}\right]\right], n \geqslant 2$, be the free associative power series ring over the integers and let $C$ be the ideal of $\mathbf{P}_{n}$ generated by all $y_{i}\left(y_{j}, y_{k}\right) y_{m}$ for all $i, j, k, m$. Set $R_{n}=\mathbf{P}_{n} / C$ and denote the generators of $R_{n}$ by $x_{1}, \ldots, x_{n}$, where $x_{i}=y_{i}+C$. As shown in [3], the group of units of $R_{n}$ is a centre-by-metabelian group, that is, satisfies the law $\left[g_{1}, g_{2} ; g_{3}, g_{4} ; g_{5}\right]=1$. Thus if $G_{n}$ is the free centre-by-metabelian group of rank $n$ with generators $f_{1}, \ldots, f_{n}$, then the map $\Theta: f_{i} \rightarrow 1+x_{i}, 1 \leqslant i \leqslant n$, can be extended to a representation of $G_{n}$ in $R_{n}$. C. K. Gupta [1] has shown that the kernel of $\Theta$ is an elementary abelian 2-group of rank $\left(\begin{array}{l}n \\ 4\end{array}\right)$ contained in the centre of $G_{n}$ for $n \geqslant 4$, but for $n=2,3$ the representation is faithful.

For any $w \in G_{n}, w \Theta$ is a power series of the form $1+\sum_{i=1}^{\infty}\langle w \Theta\rangle_{i}$, where $\langle w \Theta\rangle_{i}$ denotes the component of terms of total degree $i$. As in the corresponding representation of the free group of rank $n$ (see [4], chapter 5) the elements $w \in \gamma_{c}\left(G_{n}\right)$ are characterized by $\langle w \Theta\rangle_{i}=0, i \leqslant c-1$. Thus, an element $w \in$ $\gamma_{c}\left(G_{n}\right)$ will be a relator for $\Phi_{c}\left(G_{n}\right)$, the $c$ th lower central factor of $G_{n}$, only if $\langle w \Theta\rangle_{c}=0$. In particular, if $w$ is a relator for $\Phi_{c}\left(G_{n}\right)$ then $w \in \gamma_{c}\left(G_{n}\right) \cap G_{n}^{\prime \prime}$ and $\langle w \Theta\rangle_{c}=0$.

The simple basic commutators of the form $\left[f_{i}, f_{j}, f_{k}, \ldots, f_{m}\right]$ of weight $c$ with $i>j \leqslant k \leqslant \cdots \leqslant m$ form a basis for $\Phi_{c}\left(G_{n} / G_{n}^{\prime \prime}\right)$ (see [5], page 106) and is a part of the basis for $\Phi_{c}\left(G_{n}\right)$. Thus if we write $\Phi_{c}\left(G_{n}\right)=F_{n, c} \times T_{n, c}$, where $F_{n, c}$ is free abelian and $T_{n, c}$ is the torsion group of $\Phi_{c}\left(G_{n}\right)$, our problem reduces to extending the simple basic commutators to a basis for $F_{n, c}$ and to finding a basis for $T_{n, c^{*}}$

\section{The structure of $\Phi_{c}\left(G_{n}\right), c$ odd, $c \geqslant 5$}

For $c \geqslant 5$, let $\omega_{c}^{*}$ and $\omega_{c}^{* *}$ be defined as

$$
\begin{aligned}
\omega_{c}^{*}= & {\left[f_{1}, f_{2} ; f_{3}, f_{4}, f_{5}, \ldots, f_{c}\right] } \\
& {\left[f_{2}, f_{3} ; f_{1}, f_{4}, f_{5}, \ldots, f_{c}\right] } \\
& {\left[f_{3}, f_{1} ; f_{2}, f_{4}, f_{5}, \ldots, f_{c}\right], }
\end{aligned}
$$


and

$$
\begin{aligned}
\omega_{c}^{* *}= & {\left[f_{1}, f_{2} ; f_{4}, f_{5}, f_{3}, f_{6}, \ldots, f_{c}\right] } \\
& {\left[f_{2}, f_{3} ; f_{4}, f_{5}, f_{1}, f_{6}, \ldots, f_{c}\right] } \\
& {\left[f_{3}, f_{1} ; f_{4}, f_{5}, f_{2}, f_{6}, \ldots, f_{c}\right] . }
\end{aligned}
$$

Then we have the following lemma.

LEMMA 1. For $c$ odd, $c \geqslant 5$,

(i) $\omega_{c}^{*}\left(G_{n}\right) \leqslant \gamma_{c+1}\left(G_{n}\right)$ for $n \geqslant c$;

(ii) $\omega_{c}^{* 2}\left(G_{n}\right) \leqslant \gamma_{c+1}\left(G_{n}\right)$ for all $n$;

(iii) $\omega_{c}^{*}\left(G_{n}\right) \leqslant \gamma_{c+1}\left(G_{n}\right)$ for $n<c$;

(iv) $\omega_{c}^{*}$ is unaltered, modulo $\gamma_{c+1}\left(G_{n}\right)$, by an arbitrary permutation of $\left\{f_{1}, \ldots, f_{c}\right\}$;

(v) $\omega_{c}^{* *}\left(G_{n}\right) \leqslant \gamma_{c+1}\left(G_{n}\right)$ for all $n$ and all $c$ even or odd.

Proof. The proofs of (i) to (iv) follow from Lemma 3.8 of [2]. For the proof of (v) we simply observe that, modulo $\gamma_{c+1}\left(G_{n}\right)$,

$$
\begin{aligned}
\omega_{c}^{* *} \equiv & {\left[f_{1}, f_{2}, f_{3} ; f_{4}, f_{5}, \ldots, f_{c}\right]^{-1} } \\
& {\left[f_{2}, f_{3}, f_{1} ; f_{4}, f_{5}, \ldots, f_{c}\right]^{-1} } \\
& {\left[f_{3}, f_{1}, f_{2} ; f_{4}, f_{5}, \ldots, f_{c}\right]^{-1}, }
\end{aligned}
$$

which is trivial by the Jacobi congruence.

We can now give the structure of $\Phi_{c}\left(G_{n}\right)$ for $c$ odd, $c \geqslant 5$.

TheOREM 1. For $c$ odd, $c \geqslant 5$, let $\Phi_{c}\left(G_{n}\right)=F_{n, c} \times T_{n, c}$ where $F_{n, c}$ is free abelian and $T_{n, c}$ is the torsion subgroup. Then

(a) a basis for $T_{n, c}$ consists of the $\left(\begin{array}{c}n \\ c\end{array}\right)$ elements $\omega_{c}^{*}(k(1), \ldots, k(c))$ given by

$$
\begin{aligned}
\omega_{c}^{*}(k(1), \ldots, k(c))= & {\left[f_{k(1)}, f_{k(2)} ; f_{k(3)}, f_{k(4)}, f_{k(5)}, \ldots, f_{k(c)}\right] } \\
& {\left[f_{k(2)}, f_{k(3)} ; f_{k(1)}, f_{k(4)}, f_{k(5)}, \ldots, f_{k(c)}\right] } \\
& {\left[f_{k(3)}, f_{k(1)} ; f_{k(2)}, f_{k(4)}, f_{k(5)}, \ldots, f_{k(c)}\right], }
\end{aligned}
$$

with $1 \leqslant k(1) \leqslant \cdots \leqslant k(c) \leqslant n$;

(b) $a$ basis for $F_{n, c}$ consists of all simple basic commutators of weight $c$ with entries from the set $\left\{f_{1}, \ldots, f_{n}\right\}$ together with all commutators

$$
\left[f_{i}, f_{j} ; f_{k(1)}, f_{k(2)}, f_{k(3)}, \ldots, f_{k(c-2)}\right]
$$

with $i>j ; i \geqslant k(1) ; k(1)>k(2) \leqslant j \leqslant k(3) \leqslant \cdots \leqslant k(c-2)$. 
Proof. The simple basic commutators of weight $c$ with entries from the set $\left\{f_{1}, \ldots, f_{n}\right\}$ form a basic of $\Phi_{c}\left(G_{n} / G_{n}^{\prime \prime}\right)$ and hence constitute a part of the basis for $F_{n, c}$. Let $\omega \in \gamma_{c}\left(G_{n}\right) \cap G_{n}^{\prime \prime}$ be a relator for $\Phi_{c}\left(G_{n}\right)$. Then $\langle\omega \Theta\rangle_{c}=0$ and it follows by Lemma 4.1(iv) of [2] that if $\omega \notin \gamma_{c+1}\left(G_{n}\right)$ then $\omega$ lies in the fully invariant closure of $\omega_{c}^{*}$. The proof of (a) now follows immediately by Lemma 1((i)-(iv)). For the remainder of the proof of (b) we first observe, using Lemma 3.1((i) and (iii)) of [2], that $\omega$ can be written, modulo $\gamma_{c+1}\left(G_{n}\right)$, as a product of commutators of weight $c$ of the form

$$
z=\left[f_{i}, f_{j} ; f_{k(1)}, f_{(2)}, f_{k(3)}, \ldots, f_{k(c-2)}\right]
$$

with $i>j \geqslant k(2) ; k(1)>k(2) \leqslant k(3) \leqslant \cdots \leqslant k(c-2)$. It remains to show that modulo $\omega_{c}^{*}\left(G_{n}\right)$ we can further assume that $i \geqslant i(1)$ and $j \leqslant k(3)$ in $z$. Let $m=\max \{i, j, k(1), k(3)\}$.

Type 1 . $(z$ with $k(3)=m)$. Let $\{i, j, k(1)\}=\{a, b, c\}$ with $a \leqslant b \leqslant c$. The modulo $\omega_{c}^{*}\left(G_{n}\right), z$ can be expressed in terms of commutators $\left[f_{c}, f_{a} ; f_{b}, f_{k(1)}, f_{m}, \ldots\right]$ and $\left[f_{c}, f_{b} ; f_{a}, f_{k(1)}, f_{m}, \ldots\right]$ each of which is of the required form.

Type 2. ( $z$ with $i=m$ ). Let $\{j, k(1), k(3)\}=\{a, b, c\}$. Then $z$ is of the form $\left[f_{m}, f_{a} ; f_{b}, f_{k(1)}, f_{c}, \ldots\right]$. If $a>c$ then modulo $\omega_{c}^{* *}\left(G_{n}\right), z$ can be expressed as a product of Type 1 and the required commutator $\left[f_{m}, f_{c} ; f_{b}, f_{k(1)}, f_{a}, \ldots\right]$.

Type 3. ( $z$ with $k(1)=m$ ). Here modulo $\omega_{c}^{*}\left(G_{n}\right), z$ can be expressed as a product of Type 1 and Type 2 commutators. This completes the proof of Theorem 1.

\section{The structure of $\Phi_{c}\left(G_{n}\right), c$ even, $c \geqslant 6$}

We begin by recalling some results from [2]. For the remainder of the paper, we assume $c$ even, $c \geqslant 6$.

LeMMa 2. Let $z=\left[f_{i}, f_{j} ; f_{i}, f_{j}, p_{1} f_{1}, \ldots, p_{n} f_{n}\right]$ be an element of $\gamma_{c}\left(G_{n}\right)$, where $c=4+p_{1}+\cdots+p_{n}, p_{i} \geqslant 0$.

(i) In $R_{n}, z \Theta$ is obtained by expanding

$$
\begin{aligned}
z \Theta=1 & +\left(x_{i}, x_{j}\right) x p^{p_{1}} \cdots x_{n}^{p_{n}}\left(1+x_{i}\right)^{-1}\left(1+x_{j}\right)^{-1} \\
& \cdot\left\{\left(1+x_{1}\right)^{-p_{1}} \cdots\left(1+x_{n}\right)^{-p_{n}}-1\right\}\left(x_{i}, x_{j}\right) .
\end{aligned}
$$

(ii)

$$
\langle z \Theta\rangle_{c+1}=-\sum_{m=1}^{n} p_{m}\left(x_{i}, x_{j}\right) x_{1} p_{i} \cdots x_{m}^{p_{m+1}} \cdots x_{n}^{p_{n}}\left(x_{i}, x_{j}\right),
$$

(iii) $\left\langle z^{2} \Theta\right\rangle_{c+1}=2\langle z \Theta\rangle_{c+1}$.

(iv) If $w \in \gamma_{c}\left(G_{n}\right) \cap G_{n}^{\prime \prime}$ with $\langle w \theta\rangle_{c}=0$, then $w$ is a product of commutators of the form $z$. 
(v) Let $w$ be a product of commutators of the form $z$ with weight at least 1 in each $f_{i}$. If $\langle w \Theta\rangle_{c}=0$ and $\langle w \Theta\rangle_{c+1} \equiv 0(\bmod 2)$, then $w \in \gamma_{c+1}\left(G_{n}\right)$.

(vi) Let $\alpha_{i i}\langle w \Theta\rangle_{c}$ denote the component of $\langle w \Theta\rangle_{c}$ of those terms which begin and end with $x_{i}$. If $w \in \gamma_{c+1}\left(G_{n}\right) \cap G_{n}^{\prime \prime}$ then $\alpha_{i i}\langle w \Theta\rangle_{c+1} \equiv 0(2)$.

The proof of (i) follows from Lemma 3.4 of [2]; (ii), (iii) are easy consequence of (i); (iv) follows from Lemma 4.1(i) of [2]; (v) follows from Lemma 4.4 of [2] and (vi) follows from Corollary 3.5 of [3].

LEMMA 3. If $G$ is a centre-by-metabelian group, then for all $d \in \gamma_{m}(G), g_{i} \in G$, $k \geqslant 1, m \geqslant 2$,

$$
\left[d ; d, g_{1}, \ldots, g_{2 k}\right]^{2} \equiv \prod_{i=1}^{2 k}\left[d ; d, g_{1}, \ldots, g_{i}, g_{i}, \ldots, g_{2 k}\right]
$$

modulo $\gamma_{2 m+2 k+2}(G)$.

Proof. By Lemma 3.1 of [2], for any $d_{1}, d_{2} \in G^{\prime}, g \in G,\left[d_{1} ; d_{2}, g\right]$ $\left[d_{1}, g ; d_{2}\right]=\left[d_{1}, g ; d_{2}, g\right]^{-1}$. Further, for any $g_{i} \in G,\left[d_{1} ; d_{2}, g_{1}, g_{2}\right]=$ $\left[d_{1} ; d_{2}, g_{2}, g_{1}\right]$. Thus,

$$
\begin{aligned}
& {\left[d ; d, g_{1}, \ldots, g_{2 k}\right]} \\
& \quad=\left[d, g_{1} ; d, g_{2}, \ldots, g_{2 k}\right]^{-1}\left[d, g_{1} ; d, g_{1}, g_{2}, \ldots, g_{2 k}\right]^{-1} \\
& \quad \equiv\left[d, g_{1} ; d, g_{2}, \ldots, g_{2 k}\right]^{-1}\left[d ; d, g_{1}, g_{1}, g_{2}, \ldots, g_{2 k}\right] \\
& \quad\left(\operatorname{modulo} \gamma_{2 m+2 k+2}(G)\right)
\end{aligned}
$$

Similarly,

$$
\begin{aligned}
& {\left[d, g_{1} ; d, g_{2}, \ldots, g_{2 k}\right]^{-1}} \\
& \quad \equiv\left[d, g_{1}, g_{2} ; d, g_{3}, \ldots, g_{2 k}\right]\left[d, g_{1}, g_{2} ; d, g_{2}, g_{3}, \ldots, g_{2 k}\right] \\
& \quad \equiv\left[d, g_{1}, g_{2} ; d, g_{3}, \ldots, g_{2 k}\right]\left[d ; d, g_{1}, g_{2}, g_{2}, g_{3}, \ldots, g_{2 k}\right]
\end{aligned}
$$

Repeated applications of this step yield

$$
\begin{aligned}
& {\left[d ; d, g_{1}, \ldots, g_{2 k}\right]} \\
& \quad \equiv\left[d, g_{1}, \ldots, g_{2 k} ; d\right] \prod_{i=1}^{2 k}\left[d ; d, g_{1}, \ldots, g_{i}, g_{i}, \ldots, g_{2 k}\right] ;
\end{aligned}
$$

or equivalently,

$$
\left[d ; d, g_{1}, \ldots, g_{2 k}\right]^{2} \equiv \prod_{i=1}^{2 k}\left[d ; d, g_{1}, \ldots, g_{i}, g_{i}, \ldots, g_{2 k}\right] \text {. }
$$


Lemmas 3 and 2(ii) yield the following as a corollary.

LEMMA 4. Let $z$ be as in Lemma 2. Then $\left\langle z^{2} \Theta\right\rangle_{c}=0$ and

$$
\left\langle z^{2} \Theta\right\rangle_{c+1}=\sum_{m=1}^{n} p_{m}\left(x_{i}, x_{j} ; x_{i}, x_{j}, p_{1} x_{1}, \ldots,\left(p_{m}+1\right) x_{m}, \ldots, p_{n} x_{n}\right) .
$$

More generally, if $w=z_{1} \cdots z_{t}$ with each $z_{i}$ as above, then

$$
\left\langle w^{2} \Theta\right\rangle_{c+1}=\left\langle z_{1}^{2} \Theta\right\rangle_{c+1}+\cdots+\left\langle z_{t}^{2} \Theta\right\rangle_{c+1} .
$$

We now establish a useful criterion for identifying relations in $\Phi_{c}\left(G_{n}\right), c$ even;

Lemma 5. Let $s(w)=\left\langle w^{2} \Theta\right\rangle_{c+1}$. An element $w \in \gamma_{c}\left(G_{n}\right)$ is a relator of $\Phi_{c}\left(G_{n}\right)$ if and only if $s(w)=\left\langle v^{2} \Theta\right\rangle_{c+1}$ for some $v \in \gamma_{c+1}\left(G_{n}\right)$.

Proof. If $w$ is a relator of $\Phi_{c}\left(G_{n}\right)$ then $w \in \gamma_{c+1}\left(G_{n}\right)$ and we may choose $v=w$. Conversely, let $s(w)=\left\langle v^{2} \Theta\right\rangle_{c+1}$ for some $v \in \gamma_{c+1}\left(G_{n}\right)$. By Lemma 4, $\left\langle w^{2} \Theta\right\rangle_{c+1}=\left\langle v^{2} \Theta\right\rangle_{c+1}$ implies $2\langle\omega \Theta\rangle_{c+1}=2\langle v \Theta\rangle_{c+1}$ and, in turn, $\langle w \Theta\rangle_{c+1}$ $=\langle v \Theta\rangle_{c+1},\left\langle w v^{-1} \Theta\right\rangle_{c+1}=0$. By Lemma 2(i), $\langle w \Theta\rangle_{c}=0$ and, by hypothesis, $\langle v \Theta\rangle_{c}=0$. Thus $\left\langle w v^{-1} \Theta\right\rangle_{c}=0$, and it follows by Lemma $2(v)$ that $w v^{-1} \in$ $\gamma_{c+1}\left(G_{n}\right)$. Thus $w \in \gamma_{c+1}\left(G_{n}\right)$ as required.

As a corollary to Lemma 5 we obtain the following result:

LEMMA 6. Let $w \in T_{n, c}$ be a relator for $\Phi_{c}\left(G_{n}\right)$. Then $\alpha_{i i}(s(w)) \equiv 0(\bmod 4)$ for all $i=1, \ldots, n$.

Proof. By Lemma 5, $s(w)=\left\langle v^{2} \Theta\right\rangle_{c+1}$ for some $v \in \gamma_{c+1}\left(G_{n}\right)$. By Lemma 2(vi), $\alpha_{i i}\langle v \Theta\rangle_{c+1} \equiv 0(\bmod 2)$. Thus $\alpha_{i i}(s(w)) \equiv 0(\bmod 4)$ as required.

For convenience, we will abbreviate $z=\left[f_{i}, f_{j} ; f_{i}, f_{j}, p_{1} f_{1}, \ldots, p_{n} f_{n}\right]$ by $z=[i, j$; $\left.p_{1}, \ldots, p_{n}\right]$. Further, for any $f_{k}$ we define

$$
\left[z: q_{k} f_{k}\right]=\left[f_{i}, f_{j} ; f_{i}, f_{j}, p_{1} f_{1}, \ldots, p_{n} f_{n}, q_{k} f_{n}\right] .
$$

Using this notation we obtain the following consequence of Lemma 3.

Lemma 7. Let $z=\left[i, j ; p_{1}, \ldots, p_{n}\right]$ with $c=4+p_{1}+\cdots+p_{n}$. Then for arbitrary $k$,

$$
\left[z: 2 f_{k}\right]^{2} \equiv \prod_{i=1}^{n}\left[z: 2 f_{k}: f_{i}\right]^{p_{i}}\left[z: 3 f_{k}\right]^{2} \bmod \gamma_{c+4}\left(G_{n+1}\right)
$$


(ii)

$$
\left\langle\left[z: 2 f_{k}\right]^{2} \Theta\right\rangle_{c+3}=\left(\left\langle z^{2} \Theta\right\rangle_{c+1}: 2 x_{k}\right)+2\left\langle\left[z: 3 f_{k}\right] \Theta\right\rangle_{c+3},
$$

where $\left\langle z^{2} \Theta\right\rangle_{c+1}$ is as determined in Lemma 4.

[7(ii) is a direct consequence of 7(i).]

The next lemma gives a method for generating relations.

LEMMA 8. If $w$ is a relator for $T_{n, c}$, then $\left[w: 2 f_{k}\right]$ is a relator for $T_{n+1, c+2}$ for any $k \leqslant n+1$.

Proof. By Lemma 5 it suffices to show that $s\left(\left[w: 2 f_{k}\right]\right)=\left\langle u^{2} \Theta\right\rangle_{c+3}$ for some $u \in \gamma_{c+3}\left(G_{n+1}\right)$. Since $w$ is a relator for $T_{n, c}$, by Lemma $5 s(w)=\left\langle v^{2} \Theta\right\rangle_{c+1}$ for some $v \in \gamma_{c+1}\left(G_{n}\right)$. Thus

$$
\begin{aligned}
s\left(\left[w: 2 f_{k}\right]\right) & =\left\langle\left[w: 2 f_{k}\right]^{2} \Theta\right\rangle_{c+3} \\
& =\left(\left\langle w^{2} \Theta\right\rangle_{c+1}: 2 x_{k}\right)+2\left\langle\left[w: 3 f_{k}\right] \Theta\right\rangle_{c+3} \quad \text { (by Lemma 7) } \\
& =\left(\left\langle v^{2} \Theta\right\rangle_{c+1}: 2 x_{k}\right)+\left\langle\left[w: 3 f_{k}\right]^{2} \Theta\right\rangle_{c+3} \\
& =\left\langle\left[v: 2 f_{k}\right]^{2} \Theta\right\rangle_{c+3}+\left\langle\left[w: 3 f_{k}\right]^{2} \Theta\right\rangle_{c+3} \\
& =\left\langle u^{2} \Theta\right\rangle_{c+3},
\end{aligned}
$$

with $u=\left[v: 2 f_{k}\right]\left[w: 3 f_{k}\right] \in \gamma_{c+3}\left(G_{n+1}\right)$.

It follows from Lemma 2(iv) that for $c$ even, $c \geqslant 6$, any relator $w$ of $T_{n, c}$ is a product of commutators of the form $z=\left[i, j: p_{1}, \ldots, p_{n}\right], 4+p_{1}+\cdots+p_{n}=c$. Since $z^{2} \equiv 1 \bmod \gamma_{c+1}\left(G_{n}\right), T_{n, c}$ is, in fact, generated by all such commutators. Thus to determine the relators of $T_{n, c}$ it suffices to assume that $n \leqslant c-2$ and that each $\left[i, j ; p_{1}, \ldots, p_{n}\right]$ has weight at least one in each generator $f_{1}, \ldots, f_{n}$.

If $c \geqslant 8$ and $n=c-2$, it follows from the proof of Theorem $\mathrm{B}(\mathrm{i})$ of [2] that there is just one relator which involves all the $c-2$ variables, namely,

$$
u(c-2)=\prod_{\sigma} a(1 \sigma, 2 \sigma)
$$

where $a(i, j)=[i, j ; 1,1, \ldots, \hat{i}, \hat{j}, \ldots, 1]$ ( $\hat{k}$ indicates $k$ missing), and $\sigma$ runs over the permutations of $\{1, \ldots, c-2\}$ with $1 \sigma<2 \sigma<\cdots<(c-2) \sigma$. Hence, we may assume that $n \leqslant c-3$. For each $i, j \in\{1, \ldots, c-3\}, i<j$, we define

$$
v(c, i, j)=\prod_{m=1}^{c-5}\left[f_{i}, f_{j} ; f_{i}, f_{j}, g_{1}, \ldots, g_{m}, g_{m}, \ldots, g_{c-5}\right],
$$

where $\left\{g_{1}, \ldots, g_{c-5}\right\}=\left\{f_{1}, \ldots, \hat{f}_{i}, \ldots, \hat{f}_{j}, \ldots, f_{c-3}\right\}$. By Lemma 3.2(i) of [2], $G_{n}$ satisfies the congruence $v(c, i, j) \equiv 1$. Using this fact and Lemma 8 we can reduce the generating set for $T_{n, c}$ as follows. 
Lemma 9. Let $W=\left\{\left[i, j ; p_{1}, \ldots, p_{n}\right], i<j\right\}$ be the set of all commutators of weight $c=4+p_{1}+\cdots+p_{k}$ such that $p_{k} \leqslant 1$ for all $k<i$ and the first nonzero integer reading left to right in the sequence $p_{n}, p_{n-1}, \ldots, p_{1}$ is odd. Then $W$ is a generating set for $T_{n, c}, n \leqslant c-3, c \geqslant 8$.

Proof. The condition $i<j$ is clearly no restriction. The next condition, $p_{k} \leqslant 1$ for $k<i$ follows directly from Lemma 3.1(vii) of [2], by which for any $g, g_{i}$ in a centre-by-metabelian group $G$,

$$
\begin{aligned}
& {\left[g, g_{1} ; g, g_{1}, g_{2}, g_{3}, \ldots, g_{m}, g, g\right]} \\
& \quad \equiv\left[g, g_{1} ; g, g_{1}, g_{2}, g_{2}, g_{3}, \ldots, g_{m}\right]\left[g, g_{2} ; g, g_{2}, g_{1}, g_{1}, g_{3}, \ldots, g_{m}\right]
\end{aligned}
$$

modulo $\gamma_{m+5}(G)$. The third condition, namely, the first non-zero integer in $p_{n}, \ldots, p_{1}$ is odd, may be seen as follows. As observed above $T_{n, c}$ is spanned by all commutators $z=\left[i, j ; p_{1}, \ldots, p_{n}\right]$ with $p_{k} \leqslant 1, k<i, i<j$. Without loss of generality we may further assume that $z$ involves $f_{n-1}$ and $f_{n}$. Hence if both $p_{n-1}$ and $p_{n}$ are zero, this means that $i=n-1$ and $j=n$ so $c-2=n$. Thus we may assume that at least one of $p_{n-1}, p_{n}$ is non-zero.

Case I. $p_{n} \neq 0, p_{n}$ even. Since $G_{n}$ satisfies the congruence $v(c, i, j) \equiv 1$ defined by (2), it follows that for $p_{n} \geqslant 2$,

$$
\left[i, j ; p_{1}, \ldots, p_{n}\right]^{p_{n}-1} \prod_{m=1}^{n-1}\left[i, j ; p_{1}, \ldots, p_{m}+1, \ldots, p_{n}-1\right]^{p_{m}} \equiv 1 .
$$

However, for $p_{n}$ even $\left[i, j ; p_{1}, \ldots, p_{n}\right]^{p_{n}-1} \equiv\left[i, j ; p_{1}, \ldots, p_{n}\right]$, and the proof follows since each factor $\left[i, j ; p_{1}, \ldots, p_{m}+1, \ldots, p_{n}-1\right]$ can be expressed as a product of commutators of the required form without affecting the oddness of the occurences of $f_{n}$.

Case II. $p_{n}=0, p_{n-1}$ even. In this case $z=\left[i, n ; p_{1}, \ldots, p_{n-1}, 0\right]$ and as before the law $v(c, i, n)$ gives

$$
\left[i, n ; p_{1}, \ldots, p_{n-1}, 0\right] \prod_{m=1}^{n-2}\left[i, n ; p_{1}, \ldots, p_{m+1}, \ldots, p_{n-2}, p_{n-1}-1,0\right]^{p_{m}} \equiv 1 .
$$

Since each factor $\left[i, n ; p_{1}, \ldots, p_{m}+1, \ldots, p_{n-2}, p_{n-1}-1,0\right]$ reduces, by (3), to a product of commutators of the required form and of commutators covered by the Case I, the proof follows.

LEMMA 10. For $c \geqslant 8, n \leqslant c-3$ the set $W$ defined in Lemma 9 is a basis for $T_{n, c}$.

Proof. For $z=\left[i, j ; p_{1}, \ldots, p_{n}\right], z^{\prime}=\left[i^{\prime}, j^{\prime} ; p_{1}^{\prime}, \ldots, p_{1}^{\prime}\right]$ we define $z<z^{\prime}$ if $(i, j$, $\left.p_{1}, \ldots, p_{n}\right)<\left(i^{\prime}, j^{\prime}, p_{1}^{\prime}, \ldots, p_{n}^{\prime}\right)$ in the lexicographic ordering of the $(n+2)$-tuples. The proof of Lemma 9 shows that if $z \notin W$, then $z$ can be written as a product of elements of $W$ which are less than $z$ in the above ordering. 
By Lemma 9, $W$ generates $T_{n, c}$. Let $w=z_{1} \cdots z_{r}, z_{i} \in W$, be a relator of $T_{n, c}$ with $z_{1}<\cdots<z_{r}$. By Lemma $6, \alpha_{k k}(s(w)) \equiv 0(\bmod 4)$ for all $k$. Suppose $z_{r}=\left[i, j ; p_{1}, \ldots, p_{n}\right], p_{n}$ odd, and let $\alpha_{j j}\left(s\left(z_{r}\right)\right)=x_{j} P_{r} x_{j}$. By Lemma 2(i), $P_{r}$ has a term

$$
x_{1} \cdots x_{i-1} x_{i}^{p_{i}+2} x_{i+1}^{p_{i+1}} \cdots x_{n-1}^{p_{n-1}} x_{n}^{p_{n}+1}
$$

with coefficient $2 p_{n} \neq 0(\bmod 4)$. Since $\alpha_{j j}(s(w))=\Sigma_{k=1}^{r} \alpha_{j j}\left(s\left(z_{k}\right)\right) \equiv 0(\bmod 4)$, it follows that for some $q<r, \alpha_{j j}\left(s\left(z_{q}\right)\right)=x_{j} P_{q} x_{j}$, where $P_{q}$ has a term (4). However, $z_{q}<z_{r}$ implies $z_{q}=\left[i^{\prime}, j^{\prime} ; p_{1}^{\prime}, \ldots, p_{n}^{\prime}\right]$ with $\left(i^{\prime}, j^{\prime}, p_{1}^{\prime}, \ldots, p_{n}^{\prime}\right)<$ $\left(i, j, p_{1}, \ldots, p_{n}\right)$. If $i^{\prime}<i$ then each term in $P_{q}$ has degree at least 2 in $x_{i}^{\prime}$, so none is of the form (4). If $p_{n}^{\prime}<p_{n}$ then $p_{n}^{\prime} \leqslant p_{n}-2$ and ecah term of $P_{q}$ has degree at most $p_{n}^{\prime}+1 \leqslant p_{n}-1$ in $x_{n}$, so $P_{q}$ has no term (4). Finally, if $p_{n}^{\prime}=p_{n}$ and $p_{k}^{\prime}<p_{k}$ for some $k<n$, then every term of $P_{q}$ with degree $p_{n}+1$ in $x_{n}$ has degree $p_{i}^{\prime}<p_{k}$ in $x_{k}$, and, again $P_{q}$ does not have a term (4). Thus, if $p_{n} \neq 0$, then $\alpha_{j j}(s(w)) \neq 0$ $(\bmod 4)$, and $w$ is not a realtor of $T_{n, c}$.

Thus, we may assume that $w=z_{1} \cdots z_{r}$ is a relator of $T_{n, c}$ with $z_{r}=[i, n$; $\left.p_{1}, \ldots, p_{n-1}, 0\right], p_{n-1}$ odd. As above, if $\alpha_{n n}\left(s\left(z_{r}\right)\right)=x_{n} P_{r} x_{n}, P_{r}$ will have a term

$$
2 p_{n-1} x_{1} \cdots x_{i-1} x_{i}^{p_{i}+2} x_{i+1}^{p_{i+1}} \cdots x_{n-1}^{p_{n-1}+1} \text {, }
$$

with $2 p_{n-1} \neq 0(\bmod 4)$, and it follows, as above, that this term must occur in the expansion of some $P_{q}, q<r$. The same argument shows that this, too, is not possible. This completes the proof of the lemma.

We may summarise the above results as follows.

THEOREM 2. For $c$ even, $c \geqslant 8$, the torsion subgroup $T_{n, c}$ of $\Phi_{c}\left(G_{n}\right)$ is an elementary abelian 2-group with a basis consisting of the set $W$ of all commutators $z=\left[i, j ; p_{1}, \ldots, p_{n}\right], i<j$, with the following properties:

(a) $c=4+p_{1}+\cdots+p_{n}, p_{k} \geqslant 0$ for all $k \leqslant n$;

(b) $p_{k} \leqslant 1$ for all $k<i$;

(c) the first nonzero integer reading from left to right in $p_{n}, \ldots, p_{1}$ is odd;

(d) for any $i(1)<\cdots<i(m), m \leqslant n, W$ does not contain $[i(m-1), i(m)$; $i(1), \ldots, i(m-2)]$.

Proof. The conditions (a), (b), (c) follow directly from Lemma 10, and (d) follows from the fact that if a relator contains any part of $u(c-2)$, as defined in (1), then it must contain all of $u(c-2)$.

For $c=6$, essentially the same argument applies. However, $\left[f_{i}, f_{j} ; f_{i}, f_{j}, f_{k}, f_{k}\right]$ $\in \gamma_{7}\left(G_{4}\right)$ and as C. K. Gupta [1] has shown, $u(4) \notin \gamma_{7}\left(G_{4}\right)$. The basis for $T_{n, 6}$ is given by the following theorem. 
THEOREM 3. For $c=6, T_{n, 6}$ has a basis consisting of all commutators $\left[f_{i(1)}, f_{i(2)}\right.$; $\left.f_{i(1)}, f_{i(2)}, f_{i(3)}, f_{i(4)}\right]$, with $i(1)<i(2), i(3)<i(4)$.

As with the case for odd $c$, a basis for $F_{n, c}$ must be chosen to account for the Jacobi congruence and the above structure of $T_{n, c}$. The argument is analogous to that for odd $c$ and we omit the details. The complete description of $\Phi_{c}\left(G_{n}\right), c$ even, is given by the following theorem.

THEOREM 4. For c even, $c>6$, let $\Phi_{c}\left(G_{n}\right)=F_{n, c} \times T_{n, c}$, where $T_{n, c}$ is the torsion subgroup of $\Phi_{c}\left(G_{n}\right)$ and $F_{n, c}$ is free abelian.

(i) $A$ basis for $T_{n, c}$ is given by Theorems 2 and 3 .

(ii) $F_{n, c}$ is generated by the simple basic commutators of weight $c$ and by the commutators of the form $\left[f_{i}, f_{j} ; f_{k(1)}, f_{k(2)}, \ldots, f_{k(c-2)}\right]$ subject to $k(1)>k(2) \leqslant k(3)$ $\leqslant \cdots \leqslant k(c-2) ; i>j \leqslant k(3) ; k(2) \leqslant j$ and such that $k(1)<i$ or $k(1)>i$ and $k(2)<j<i<k(1)<k(3)<\cdots<k(c-2)$ (no repeated entry) or $k(1)=i, k(2)$ $<j$ and if $k(l)$ is another repeated entry $(3 \leqslant l \leqslant c-2)$, then $k(1) \leqslant k(l)$.

CONCLUding REMARKs. With the aid of Lemma 8 we can give a different description of $T_{n, c}, c$ even, as follows.

THEOREM 5. For c even, $c \geqslant 6$, the torsion subgroup $T_{n, c}$ of $\Phi_{c}\left(G_{n}\right)$ is generated by all commutators $z=\left[i, j ; p_{1}, \ldots, p_{n}\right]$ with $4+p_{1}+\cdots+p_{n}=c, p_{i} \geqslant 0$, subject only to the relations

$\left[u\left(c^{\prime}-2\right): 2 k_{1} f_{1}: \cdots: 2 k_{n} f_{n}\right]=1$ and $\left[v\left(c^{\prime}, i, j\right): 2 k_{1} f_{1}: \cdots: 2 k_{n} f_{n}\right]=1$, where $c=c^{\prime}+2 k_{1}+\cdots+2 k_{n}, k_{l} \geqslant 0$ and $u\left(c^{\prime}-2\right), v\left(c^{\prime}, i, j\right)$ are as defined by (1) and (2).

\section{References}

1. Chander Kanta Gupta, 'The free centre-by-metabelian groups', J. Austral. Math. Soc. 16 (1973), 294-299.

2. Narain Gupta and Frank Levin, 'Separating laws for free centre-by-metabelian nilpotent groups', Comm. Algebra 4 (1976), 249-270.

3. T. C. Hurley, 'Representations of some relatively free groups in power series rings', Proc. London Math. Soc. (3) 24 (1972), 257-294.

4. Wilhelm Magnus, Abraham Karrass and Donald Solitar, Combinatorial group theory (Interscience, New York, 1966).

5. Hanna Neumann, Varieties of groups (Springer-Verlag, New York, 1967).

6. J. N. Ridley, 'The free centre-by-metabelian group of rank two', Proc. London Math. Soc. (3) 20 (1970), 321 -347. 
7. A. L. Šmelkin, 'Free polynilpotent groups', Izv. Akad. Nauk SSSR. Ser. Mat. 28 (1964), 91-122; English translation: Amer. Math. Soc. Transl. (2) 55 (1966), 270-304.

8. M. A. Ward, 'Basic commutators', Philos. Trans. Roy. Soc. London Ser. A 264 (1969), 343-412.

University of Manitoba

Winnipeg

Manitoba R3T 2N2

Canada

\section{Ruhr Universität}

4630 Bochum

West Germany
University College

Galway

Ireland 